This is the author's manuscript for publication. The publisher-formatted version may be available through the publisher's web site or your institution's library.

\title{
Physical activity intervention effects on perceived stress in working mothers: the role of self-efficacy
}

Emily L. Mailey \& Edward McAuley

How to cite this manuscript

If you make reference to this version of the manuscript, use the following information:

Mailey, E. L., \& McAuley, E. (2014). Physical activity intervention effects on perceived stress in working mothers: The role of self-efficacy. Retrieved from http://krex.ksu.edu

\section{Published Version Information}

Citation: Mailey, E. L., \& McAuley, E. (2014). Physical activity intervention effects on perceived stress in working mothers: The role of self-efficacy. Women \& Health, 54(6), 552-568.

Copyright: Copyright $\odot$ Taylor \& Francis Group, LLC

Digital Object Identifier (DOI): doi:10.1080/03630242.2014.899542

Publisher's Link:

http://www.tandfonline.com/doi/full/10.1080/03630242.2014.899542\#.VEglqcVdVQx

This item was retrieved from the K-State Research Exchange (K-REx), the institutional repository of Kansas State University. K-REx is available at http://krex.ksu.edu 
Physical activity intervention effects on perceived stress in working mothers: The role of selfefficacy

Emily L. Mailey ${ }^{\mathrm{a} 1}$ \& Edward McAuley ${ }^{\mathrm{a}}$

${ }^{a}$ Department of Kinesiology \& Community Health, University of Illinois at Urbana-Champaign, Freer Hall, Urbana, IL, 61801

${ }^{1}$ Present address: Emily L. Mailey, Kansas State University, Department of Kinesiology, 8D

Natatorium, Manhattan, KS 66506, 785-532-7287

Address all correspondence to:

Emily Mailey

Department of Kinesiology

Kansas State University

8D Natatorium

Manhattan, KS 66506

Phone: 785-532-7287

Email: emailey@ksu.edu 


\begin{abstract}
Working mothers often report elevated stress, and efforts to improve their coping resources are needed to buffer the detrimental effects of stress on health. This study examined the impact of changes in physical activity, self-efficacy, and self-regulation across the course of a brief intervention on subsequent levels of stress in working mothers. Participants $(N=141)$ were randomly assigned to an intervention or control condition (2:1 ratio). The intervention was conducted in Illinois between March 2011-January 2012 and consisted of two group-mediated workshop sessions with content based on Social Cognitive Theory. Participants completed measures of physical activity, self-efficacy, self-regulation, and perceived stress at baseline, immediately post-intervention, and 6-month follow-up. Stress levels declined across the 6-month period in both groups. Changes in stress were negatively associated with changes in self-efficacy and self-regulation among intervention participants only. Regression analyses revealed the intervention elicited short-term increases in physical activity, self-efficacy, and self-regulation, but only changes in self-efficacy predicted perceived stress at 6-month follow-up. These results suggest that enhancing self-efficacy is likely to improve working mothers' perceived capabilities to cope with stressors in their lives. Future interventions should continue to focus on increasing self-efficacy to promote improvements in physical activity and psychological well-being in this population.
\end{abstract}

Keywords: working mothers; physical activity; self-efficacy; stress 


\section{Background}

Although over $70 \%$ of mothers now work outside the home, their commitment to other more “traditional" female duties, such as childcare and household responsibilities, has not decreased proportionately. In fact, data from the Bureau of Labor Statistics (2008) show married mothers spend significantly more time doing household activities and providing childcare than married fathers. This so-called "second shift" phenomenon clearly puts significant demands on women's time, and ample evidence has suggested that the demands of fulfilling multiple roles can contribute to increased stress and anxiety among working mothers. For example, one classic study of white-collar workers found levels of stress hormones (i.e., norepinephrine and cortisol) decreased after 5:00 p.m. among men, but increased in women (Frankenhaeuser et al., 1989). Subsequently, others have demonstrated that working women with children produce significantly more cortisol over the course of a day than their childless counterparts, and that women with both work and childcare obligations report greater psychological stress than both female employees without children and male employees with children (Bekker et al., 2000; Luecken et al., 1997). Such findings are of concern given the well-documented adverse health effects of stress, including negative effects on immune function, cardiovascular health, the gastrointestinal system, and mental health (Larzelere and Jones, 2008).

Stress is produced by events perceived to be uncontrollable and threatening, such that situations that place high demands on an individual when her perceptions of control are low are most likely to induce stress (Hansen et al., 2010). Thus, it is important to note that a woman's perception of her work and family roles may be a better predictor of stress than the actual number or extent of demands. In particular, circumstances that diminish perceptions of control (e.g., frustration with partner's family role, dissatisfaction with childcare arrangements) are 
likely to trigger increases in stress, whereas having appropriate resources for coping with stress (e.g., high resourcefulness, spousal support) may attenuate adverse health effects (Rosenbaum and Cohen, 1999; Tinger et al., 1996). Given the evidence that effectively coping with stress might lessen the negative effects of multiple roles on mental health, it would be prudent to identify effective means for handling stress.

A considerable body of literature has suggested that physical activity is associated with reductions in stress and improvements in quality of life. For example, data from the Copenhagen City Heart Study showed a significant decrease in the likelihood of an individual reporting high stress as self-reported level of physical activity increased (Schnohr et al., 2005). Among working mothers, research examining the relationship between physical activity and stress has been sparse, but some evidence from related populations has suggested that physical activity might be an effective means for self-regulating stress and anxiety within this group. An early intervention for stressed working women found exercise to be as effective as progressive relaxation for reducing anxiety and increasing problem-focused coping (Long and Haney, 1988). More recently, increases in physical activity across a 10-week intervention period were associated with decreases in self-reported stress in a sample of low-income mothers (Urizar et al., 2005). Finally, women caring for a spouse with dementia evidenced significant reductions in perceived stress after participating in a 6-month telephone-delivered intervention to promote physical activity (Connell and Janevic, 2009).

Unfortunately, many working mothers are not enjoying the physical and mental health benefits of physical activity. As a group, working mothers exhibit high levels of inactivity, which can largely be attributed to the numerous real and perceived physical activity barriers they encounter (Brown et al., 2001; Burke et al., 2004; Cramp and Bray, 2011; Verhoef and Love, 
1994). As declining levels of physical activity across the transition into motherhood have emerged as a legitimate public health concern, several interventions designed specifically for women with children have been developed (Cody and Lee, 1999; Cramp and Brawley, 2006; Fahrenwald et al., 2004; Fjeldsoe et al., 2010). Most recently, a brief, social cognitive theorybased intervention to increase physical activity among working mothers demonstrated positive effects on self-reported physical activity (Mailey and McAuley, 2013). Five months after completion of the brief intervention, increases in physical activity were sustained and shown to be mediated by changes in self-efficacy and self-regulation. From a theoretical standpoint, increases in self-efficacy (i.e., confidence in one's capabilities to execute a specific course of action) and self-regulation (i.e., guiding one's own actions by setting personal goals and planning courses of action to achieve them) are likely to foster increased perceptions of control over one's behavior, which could help individuals cope with stress more effectively. In fact, Bandura (1998) has been quite explicit in describing the relationship between self-efficacy and stress, asserting that stress reactions are determined by one's perceived inefficacy to exercise control over environmental demands.

Thus, the purpose of the present study was to examine the impact of changes in physical activity, self-efficacy, and self-regulation across the course of a brief behavior change intervention on subsequent levels of stress in a sample of working mothers. Although improving health and quality of life within this population is an important public health priority, few interventions to address these issues have been developed or evaluated in working women. This intervention was brief in nature and thus would have the potential to be consolidated into a training manual and disseminated if meaningful effects on key health outcomes such as stress were observed. We hypothesized that changes in physical activity, self-efficacy, and self- 
regulation elicited by the intervention would enhance participants' coping capacity and thus significantly contribute to reductions in perceived stress five months after completion of the intervention.

\section{Methods}

The present study reports a secondary analysis of an intervention whose primary outcomes have been previously published (Mailey and McAuley, 2013). Because stress is a critical health outcome among working mothers, the extent to which a brief social cognitive theory-based physical activity intervention could elicit reductions in stress was an important question to be addressed.

\section{Participants}

Participants $(N=141)$ were females aged 25-52 who were employed at least 25 hours per week and had at least one child under age 15 living at home. They were recruited via local moms groups, day care centers, and various media outlets (e.g., email lists, newspaper, radio). To be considered for the study, participants had to be willing to be randomized, able to attend two workshop sessions, and able to access the internet. Women who were already meeting or exceeding the national physical activity recommendations (i.e., more than 150 minutes of moderate activity per week for the previous 2 months) based on self-reported activity levels were excluded. Individuals who expressed interest in participating in the study completed a brief prescreening questionnaire to assess for eligibility via telephone or email; those who met all inclusion criteria were subsequently sent instructions for completing the baseline questionnaires.

\section{Measures}




\section{Demographics}

We administered a demographics questionnaire to ascertain participants' age, race, education, income, marital status, employment status, and parenthood status (including number and ages of children).

\section{Physical activity}

We assessed physical activity using the Godin Leisure-Time Exercise Questionnaire (Godin and Shephard, 1985). This brief measure asks participants to report the current frequency of engaging in strenuous (e.g., running), moderate (e.g., easy bicycling or swimming), and light (e.g., bowling or golf) exercise for at least 15 minutes per session during a typical week. We calculated a total weekly leisure activity score by multiplying the frequencies of strenuous, moderate, and light activities by nine, five, and three, respectively, and then summing the products. This measure is widely used and has previously demonstrated adequate test-retest reliability and concurrent validity with objective measures of physical activity and energy expenditure (Jacobs et al., 1993).

\section{Self-efficacy}

The Barriers Self-Efficacy Scale (McAuley, 1992) assesses participants' perceived capabilities to exercise regularly in the face of commonly identified barriers to participation (e.g., bad weather, schedule conflicts), For each of the 13 items, participants responded by indicating their confidence to execute the given behavior on a 100-point percentage scale range from $0 \%$ (not at all confident) to $100 \%$ (highly confident). Total strength of self-efficacy was determined by calculating the mean of all items, resulting in a maximum possible efficacy score of 100,with 
higher scores reflecting greater self-efficacy to overcome common barriers (range: 0-100). Internal consistency of this scale was excellent ( $a=.91-.94)$.

\section{Self-regulation}

The Exercise Planning and Scheduling Scale (Rovniak et al., 2002) includes ten items related to scheduling and planning exercise as part of one's daily routine (e.g., "I schedule my exercise at specific times each week."). For each item, participants responded on a scale from 1 (does not describe) to 5 (describes completely). Responses were summed to yield a total score. Higher scores reflect greater use of self-regulatory strategies (range: 10-50). Internal consistency was $\operatorname{good}(a=.80-.90)$.

\section{Stress}

The Perceived Stress Scale (Cohen et al., 1983) is a 10-item scale designed to tap general beliefs about perceived stress. It assesses stress appraisals from a transactional perspective, in which the extent to which events are perceived to be uncontrollable and threatening determines the stress response (e.g., "In the last month, how often have you found that you could not cope with all the things that you had to do?"). Higher scores reflect greater perceived stress (range: 0-40). Internal consistency for this measure in the present study was good ( $a=.88-.92)$.

\section{Procedures}

All procedures were approved by a University Institutional Review Board. Participant recruitment began in March 2011, and follow-up data collection was complete in January 2012. All participants signed an informed consent document prior to being included in the study. The full study procedures have been previously reported elsewhere (Mailey and McAuley, 2013). Briefly, participants who met inclusion criteria and agreed to participate were emailed a link to 
complete the questionnaires online. Once an individual's baseline data was received, she was randomly assigned to an intervention $(n=95)$ or waitlist control $(n=46)$ condition. An independent investigator conducted the randomization using a computerized data management system. Participants were randomized at a 2:1 ratio because the initial study was designed to determine whether telephone support during the follow-up period would improve long-term outcomes. Because the two intervention groups did not exhibit differential changes on any of the measured outcomes, they were combined for the present investigation. All participants completed the questionnaires again immediately post-intervention (one month), and six months after baseline. After all six-month follow-up data had been collected, we invited all waitlist control participants to receive the intervention.

The intervention took place during the first month following randomization and consisted of two interactive group-based sessions, spaced three weeks apart, which taught participants behavior modification strategies based on social cognitive principles. For example, participants identified their primary barriers and developed strategies for overcoming them, set specific physical activity goals, and brainstormed ideas for fitting in physical activity throughout the day. The intervention targeted self-efficacy by encouraging participants to focus on realistic shortterm objectives, asking them to share their "success stories" with the group, discussing how confidence in one's capabilities can improve adherence, and showing them video clips of active local working moms describing their strategies for making time for physical activity. We also encouraged participants to broaden their definitions of "exercise" to include activities that can be completed at home, are moderate in intensity, and are as brief as 10 minutes in duration. Individuals attended the 2-hour workshop sessions in groups with approximately 6-8 other moms, and the sessions were interactive and incorporated small and large group discussions and 
problem-solving activities. All participants received a pedometer, a written social cognitive theory-based handbook, and a 1-hour individual session with a personal trainer. For a detailed description of the intervention content, see Mailey and McAuley (2013).

We intentionally designed the intervention to minimize face-to-face contact to maximize opportunities for participant recruitment and retention. Working mothers are unlikely to be willing or able to devote a significant amount of their time to a behavior change program; therefore this intervention sought to implement an efficacious program while being mindful of the significant time barriers faced by the target population. In addition, the brief format of the intervention would increase the likelihood that the program could be adopted and implemented in a variety of settings to have a significant public health impact.

\section{Statistical Analysis}

We used the multiple imputation analysis option in SPSS v.20 to impute missing values for participants who had discontinued participation at one and six months follow-up; all others completed all questionnaires. We pooled five imputed data sets to compute the final imputed values. Missing data ranged from $19.9 \%$ at one-month follow-up to $24.8 \%$ at the six-month follow-up. We repeated all analyses using the original data set with missing data and findings were not substantially different from those conducted with the imputed data. The imputed sample results are reported herein.

To determine whether the intervention had a direct impact on participants' perceived stress across the six-month period, we conducted a 2 (Group) x 3 (Time) repeated measures ANOVA. 
To determine whether changes in perceived stress were associated with changes in physical activity, self-efficacy, or self-regulation across the six-month period, we calculated standardized residual change scores using the baseline and follow-up values for each variable, and then conducted correlation analyses to examine these relationships within the intervention and control groups separately.

Next, we conducted a series of analyses to examine whether any short-term changes in physical activity, self-efficacy, or self-regulation were associated with perceived stress at sixmonth follow-up. First, we calculated standardized residual change scores to reflect changes in physical activity, self-efficacy, and self-regulation across the brief intervention period (i.e., baseline to post-intervention). Next, we regressed changes in physical activity, self-efficacy, and self-regulation on treatment group assignment to determine whether the intervention had a significant impact on these variables (Models 1-3). Demographic variables (i.e., age, race, education, marital status, number of children, age of youngest child) were included in the models as covariates and analyses controlled for baseline levels of the dependent variables. Finally, perceived stress at follow-up was regressed on group assignment, changes in physical activity/self-efficacy/self-regulation, and the demographic variables to determine whether perceived stress at follow-up could be explained by any of these factors (Models 4-6). These analyses also controlled for baseline levels of perceived stress.

\section{Results}

\section{Participant Characteristics and Retention}

Full details of the sample, including a CONSORT diagram, have been reported previously (Mailey and McAuley, 2013). Of the 224 individuals who initially expressed interest in the 
study, 194 were screened for eligibility and 156 (80.4\%) met all inclusion criteria. Of these, 141 (90.4\%) provided complete baseline data and were randomized. On average, participants were 37.3 years old and had two children (range: 1-7) (Table 1). The average age of the youngest child was 4.75 years old (range: 2 months-15 years). A majority of participants were white, married, and working full-time. As a whole, the sample was well-educated and relatively affluent.

Of the 141 randomized participants, 119 participated in post-intervention data collection, and 109 participated in the 6-month follow-up. The most common reasons for withdrawal were schedule conflicts and family/personal matters. T-tests conducted to assess baseline differences between those who dropped out and those who completed the study revealed participants who completed the study were more educated $(t(139)=2.93, p=.005)$ than those who dropped out. No significant baseline differences were observed for any other variables.

\section{Intervention Effects on Physical Activity, Self-Efficacy, and Self-Regulation}

The effects of the intervention on physical activity and social cognitive determinants have been reported elsewhere (Table 2) (Mailey \& McAuley, 2013). Briefly, intervention participants exhibited a significantly larger increase in self-reported physical activity than control participants immediately following the brief intervention. Among intervention participants, physical activity levels declined slightly across the follow-up period, but they remained more active than participants assigned to the control group. Self-efficacy increased initially within the intervention group only, but decreased significantly from post-intervention to six-month follow-up. Selfreported use of self-regulatory strategies did not change across the six-month period within the control group, but increased significantly within the intervention group. Long-term changes in physical activity were mediated by changes in self-efficacy and self-regulation. 


\section{Intervention Effects on Stress}

In examining the effects of the intervention on perceived stress, the ANOVA revealed a significant main effect for time $\left[F(2,138)=10.35, p<.001, \eta^{2}=0.13\right]$. Both groups reported decreases in stress across the 6-month period (Table 2). The interaction effect was not significant $\left[F(2,138)=0.63, p=.53, \eta^{2}=0.01\right]$, indicating that changes in stress did not differ as a function of treatment group assignment.

\section{Relationships Among Changes in Perceived Stress, Physical Activity, Self-Efficacy, and Self-Regulation}

Correlation analyses revealed changes in perceived stress were significantly associated with changes in self-efficacy $(r=-.44, p<.001)$ and self-regulation $(r=-.34, p=.001)$ among participants assigned to the intervention condition, such that increases in self-efficacy and self-regulation were related to decreases in perceived stress. Among participants who received the intervention, decreases in stress were associated with increased physical activity and approached significance $(r=-.19, p=.07)$. Within the control condition, changes in perceived stress across the six-month period were not significantly associated with changes in physical activity $(r=.15, p=.31)$, selfefficacy $(r=-.21, p=.16)$, or self-regulation $(r=.19, p=.22)$.

Are intervention effects on stress explained by changes in physical activity, self-efficacy, or self-regulation?

Controlling for demographic factors and baseline levels of each respective variable, regression analyses revealed treatment group assignment predicted changes in physical activity (Model 1; $\beta=.284, p=.001)$, self-efficacy (Model 2; $\beta=.226, p=.01$ ), and self-regulation (Model 3; $\beta=.388, p<.001$ ) across the intervention period (Table 3). 
Next, we conducted three additional regression analyses to test models 4-6 (Figure 1). Changes in physical activity (Model 4; $\beta=.110, p=.16$ ) and self-regulation (Model 6; $\beta=-.064$, $p=.439$ ) did not significantly contribute to perceived stress at the six-month follow-up. Changes in self-efficacy, however, were a significant independent predictor of perceived stress at followup (Model 5; $\beta=-.256, p=.001)$ (Figure 1).

\section{Discussion}

The demands associated with fulfilling multiple roles often lead to elevated levels of stress among working mothers (Bekker et al., 2000; Luecken et al., 1997), and prolonged stress is associated with numerous negative physical and mental health outcomes (Larzelere and Jones, 2008). Increasing individuals' ability to cope with stress is a useful approach for buffering the detrimental effects of stress on health. This study explored these issues in the context of a social cognitive theory-based intervention designed to increase physical activity among working mothers. We hypothesized that increases in physical activity, self-efficacy, and self-regulation acquired as a function of participation in the intervention would serve as resources for coping with future stress.

Across the six-month period, all participants, regardless of treatment group assignment, reported reductions in perceived stress. Although we had anticipated that the intervention would elicit reductions in stress, it was somewhat surprising that individuals assigned to the control condition exhibited comparable reductions. Interestingly, changes in stress among intervention participants, but not control participants, were associated with changes in self-efficacy and selfregulation. This suggests that the cognitive and behavioral skills acquired through intervention participation may have been utilized as a means of regulating negative emotional states. As 
changes in stress within the control group were unrelated to any of the constructs measured, any explanations for these results are purely speculative. It is possible that stress reductions among these participants were a function of seasonal effects, the Hawthorne effect (i.e., reactivity to being measured), or a social desirability bias.

Based on previous research, we hypothesized increases in physical activity would be a key predictor of perceived stress. Various physiological and psychological explanations for the stress-relieving effects of physical activity (e.g., release of endorphins, distraction from daily worries) have been tested and received sufficient support in the physical activity literature (Breus and O’Connor, 1998; Jarvekulg and Viru, 2002). However, as this intervention was based on social cognitive theory, an important objective was to determine whether self-efficacy and/or self-regulatory skills acquired as a function of intervention participation might also play a role. Results showed changes in self-efficacy, but not physical activity or self-regulation, significantly predicted future levels of perceived stress. These findings are consistent with results of previous interventions that have found health outcomes are more strongly related to one's self-regulatory efficacy than to the health behaviors themselves. For example, females who received behavioral counseling as part of the Activity Counseling Trial reported reductions in perceived stress 24 months later that were mediated by barriers self-efficacy (Anderson et al., 2005). The authors of this study pointed out that the benefits of cognitive and behavioral skills acquired during interventions are often underemphasized, but may indeed be driving changes in health outcomes such as stress.

Self-efficacy has consistently emerged as a mediator of intervention effects on health outcomes (e.g., psychological well-being, function/disability, quality of life) in a variety of populations (Elavsky et al., 2005; Lorig et al., 2006; McAuley et al., 2007; McAuley et al., 
2008). In particular, the relationship between stress and self-efficacy has been studied extensively. The results of this study complement existing literature that highlights the role of self-efficacy in the stress appraisal process. Although the barriers self-efficacy scale used in this study was specific to physical activity, it could be viewed as a measure of coping self-efficacy that more broadly reflects individuals' confidence in their ability to develop effective strategies and invest effort to overcome the obstacles they encounter. Whereas individuals with low coping efficacy are likely to dwell on their failures and stall their efforts to move forward, more efficacious individuals are likely to use problem-solving strategies to generate a variety of coping strategies for overcoming difficulties (Bandura, 1982). This type of problem-focused coping has been associated with an increased sense of control over one's environment, leading individuals to develop skills for dealing with daily stressors and employ efforts to change the environment to reduce the likelihood that they will encounter similar stress-inducing events in the future (Chwalisz et al., 1992).

Although self-efficacy appears to be a key mediator of behavioral and health outcomes, developing interventions to elicit and sustain improvements in self-efficacy remains an ongoing challenge. In the present study, initial increases in self-efficacy across the intervention period were not sustained at the 6-month follow-up (Mailey and McAuley, 2013). Such declines are not uncommon in the context of physical activity interventions and reflect the unstable nature of efficacy judgments (McAuley et al., 2011). Furthermore, as a group, participants reported remarkably low levels of self-efficacy (35-50\%), which highlights the extent to which numerous barriers make regular exercise a significant challenge among working mothers. Future interventions targeting this population will need to devote substantial attention to creating mastery experiences and practicing problem-focused coping to develop an array of strategies for 
overcoming barriers. Additional plans for enhancing maintenance are also warranted and could focus on social support as key facilitator of physical activity adherence. Working mothers are likely to feel more efficacious if they have family and friends who are committed to helping them achieve their goals (Albright, Maddock, \& Nigg, 2005; Brown et al., 2001; Miller, Trost, \& Brown, 2002).

\section{Study Strengths and Limitations}

The results of this study must be understood within the context of its strengths and limitations. Working mothers make up a large, but often overlooked, segment of our population for whom high levels of stress may threaten physical and mental health. A notable strength of this study was that it examined stress reduction in the context of a theory-based randomized controlled trial. The longitudinal design of the study allowed for the testing of a prospective model in which changes in behavioral and theoretical variables temporally preceded subsequent ratings of perceived stress. The results suggested that even small, short-term improvements in self-efficacy may help working mothers cope with stress up to six months later. Several limitations must also be addressed. Participants were highly educated and relatively affluent; thus the extent to which these findings can be generalized to all working mothers is questionable. In addition, the small sample size may have provided inadequate statistical power to detect some meaningful associations as statistically significant. The study utilized a wait list control group, which has been hypothesized to attenuate naturally occurring improvements in the context of randomized controlled trials (Mohr et al., 2009). In the present study, however, the control group actually evidenced reductions in stress that were unexpected and unrelated to changes in physical activity, self-efficacy, and self-regulation. We did not collect data on other stress management techniques that might have provided an alternative explanation for these findings. 


\section{Conclusions}

Working mothers make up an increasingly large segment of the population, and strategies to reduce stress and improve quality of life are urgently needed. The results of this study showed a brief social cognitive theory-based intervention elicited improvements in self-efficacy which were related to subsequent reductions in perceived stress. Enhancing self-efficacy is likely to improve working mothers' capabilities to exercise control over stressors in their lives. Future interventions should continue to target self-efficacy in order to promote improvements in physical activity and psychological well-being in this population.

\section{Acknowledgements}

This study was supported by the Coca-Cola Company Doctoral Student Grant on Behavior Research Fund and the Raymond and Rosalee Weiss Research Endowment from the American College of Sports Medicine Foundation. 
Table 1. Participant demographics at baseline

\begin{tabular}{lcc}
\hline Variable & $\begin{array}{c}\text { Mean (SD)/Freq (\%) } \\
\text { Intervention }\end{array}$ & Control \\
\hline Age (years) & $37.75(6.91)$ & $36.35(6.06)$ \\
Number of children & $1.86(0.97)$ & $2.04(1.03)$ \\
Age (years) of youngest child & $5.01(4.01)$ & $4.22(3.49)$ \\
Employment status & & \\
$\quad$ Full-time & $87(91.6 \%)$ & $38(82.6 \%)$ \\
$\quad$ Part-time & $8(8.4 \%)$ & $8(17.4 \%)$ \\
Hours worked per week & $40.23(6.80)$ & $39.09(6.75)$ \\
Marital Status & & \\
$\quad$ Married & $77(81.1 \%)$ & $42(91.3 \%)$ \\
Divorced/separated & $10(10.5 \%)$ & $2(4.3 \%)$ \\
Partnered/significant other & $4(4.2 \%)$ & $1(2.2 \%)$ \\
$\quad$ Single & $4(4.2 \%)$ & $1(2.2 \%)$ \\
Race & & \\
$\quad$ White & $75(78.9 \%)$ & $38(82.5 \%)$ \\
African American & $9(9.5 \%)$ & $4(8.7 \%)$ \\
Asian & $9(9.5 \%)$ & $1(2.2 \%)$ \\
Other & 0 & $2(4.4 \%)$ \\
Not disclosed & $2(2.1 \%)$ & $1(2.2 \%)$ \\
Education & & \\
$\quad$ College Graduate & $12(12.6 \%)$ & $6(13.0 \%)$ \\
College Graduate & $36(37.9 \%)$ & $16(34.8 \%)$ \\
Advance Degree & $47(49.5 \%)$ & $24(52.2 \%)$ \\
Annual Household Income & & \\
<\$40,000 & $14(14.8 \%)$ & $5(10.8 \%)$ \\
$>\$ 40,000$ & $78(82.1 \%)$ & $41(89.2 \%)$ \\
Not disclosed & $3(3.2 \%)$ & 0 \\
\hline
\end{tabular}

Note. There were no significant baseline differences between participants in the intervention and control groups 
Table 2. Intervention effects on mean (standard deviation, SD) scores for physical activity, selfefficacy, self-regulation, and perceived stress

\begin{tabular}{|l|l|c|c|c|c|c|}
\hline & & 1. Baseline & 2. Post-intervention & 3. Follow-up & $d$ & $d$ \\
& & Mean (SD) & Mean (SD) & Mean (SD) & $(1-2)$ & $(1-3)$ \\
\hline Physical Activity & Intervention & $19.01(17.1)$ & $35.51(18.5)$ & $33.75(22.5)$ & .93 & .74 \\
\cline { 2 - 7 } & Control & $16.92(19.3)$ & $24.44(16.2)$ & $25.31(18.3)$ & .42 & .45 \\
\hline \multirow{2}{*}{$\begin{array}{l}\text { Barriers Self-efficacy } \\
\text { (Range: 0-100) }\end{array}$} & Intervention & $45.3(18.0)$ & $48.1(19.0)$ & $41.8(18.4)$ & .15 & -.19 \\
\cline { 2 - 8 } & Control & $40.6(20.3)$ & $37.4(22.5)$ & $34.5(23.0)$ & -.15 & -.28 \\
\hline Planning/Scheduling & Intervention & $16.1(5.63)$ & $24.5(7.69)$ & $22.1(7.79)$ & 1.25 & .88 \\
\cline { 2 - 8 } & Control & $16.0(5.89)$ & $18.4(7.12)$ & $17.6(7.06)$ & .37 & .25 \\
\hline Perceived Stress & Intervention & $17.6(6.71)$ & $14.9(6.74)$ & $15.6(7.06)$ & -.40 & -.29 \\
\cline { 2 - 8 } (Range: 0-40) & Control & $17.9(5.83)$ & $16.1(7.32)$ & $15.7(7.11)$ & -.27 & -.34 \\
\hline
\end{tabular}

Note. 1-2=Effect size (Cohen's d) for change from baseline to post-intervention; 1-3=Effect size (Cohen's d) for change from baseline to follow-up. Physical activity, barriers selfefficacy, and planning/scheduling data have been previously published (Mailey \& McAuley, 2013). 
Table 3. Regression analyses to determine whether intervention effects on stress could be explained by changes in physical activity, self-efficacy, or self-regulation

\begin{tabular}{|c|c|c|c|c|c|}
\hline Model & $F$-value & Adjusted $R^{2}$ & Standardized $\beta$ & $\mathrm{SE}$ & $t$-value \\
\hline Model $1(\mathrm{df}=8,132)$ & 1.79 & 0.04 & & & \\
\hline \multicolumn{6}{|l|}{$\Delta$ Physical activity } \\
\hline Intervention & & & .284 & .179 & $3.36 * *$ \\
\hline Physical activity $(\mathrm{m} 0)$ & & & -.035 & .005 & -0.407 \\
\hline Model $2(\mathrm{df}=8,132)$ & 1.06 & 0.003 & & & \\
\hline \multicolumn{6}{|l|}{$\Delta$ Self-efficacy } \\
\hline Intervention & & & .226 & .183 & $2.61 *$ \\
\hline Self-efficacy $(\mathrm{m} 0)$ & & & -.043 & .005 & -0.50 \\
\hline Model $3(\mathrm{df}=8,132)$ & 3.59 & 0.13 & & & \\
\hline \multicolumn{6}{|l|}{$\Delta$ Self-regulation } \\
\hline Intervention & & & .388 & .170 & $4.82 * * *$ \\
\hline Self-regulation (m0) & & & -.037 & .014 & -0.44 \\
\hline Age & & & -.258 & .018 & $-2.11 *$ \\
\hline Model $4(\mathrm{df}=9,131)$ & 5.62 & 0.23 & & & \\
\hline \multicolumn{6}{|l|}{ Perceived stress (m6) } \\
\hline Intervention & & & -.012 & 1.18 & -0.15 \\
\hline$\Delta$ Physical activity & & & .110 & .556 & 1.40 \\
\hline Stress $(\mathrm{m} 0)$ & & & .454 & .083 & $6.04 * * *$ \\
\hline Education & & & -.212 & .635 & $-2.56^{*}$ \\
\hline Model $5(\mathrm{df}=9,131)$ & 7.15 & 0.28 & & & \\
\hline \multicolumn{6}{|l|}{ Perceived stress (m6) } \\
\hline Intervention & & & .076 & 1.12 & 1.01 \\
\hline$\Delta$ Self-efficacy & & & -.256 & .522 & $-3.47 * *$ \\
\hline Stress $(\mathrm{m} 0)$ & & & .446 & .079 & $6.19 * * *$ \\
\hline Education & & & -.211 & .610 & $-2.65 * *$ \\
\hline Model $6(\mathrm{df}=9,131)$ & 5.41 & 0.22 & & & \\
\hline \multicolumn{6}{|l|}{ Perceived stress (m6) } \\
\hline Intervention & & & .044 & 1.24 & 0.53 \\
\hline$\Delta$ Self-regulation & & & -.064 & .583 & -0.78 \\
\hline Stress $(\mathrm{m} 0)$ & & & .440 & .083 & $5.85 * * *$ \\
\hline Education & & & -.199 & .637 & $-2.39 *$ \\
\hline
\end{tabular}

$* \mathrm{P}<.05 * * \mathrm{p}<.01 * * * \mathrm{p}<.001$

Note: Demographics included in all models were age, number of children, age of youngest child, marital status, race, and education. Only significant demographics are included in the table. 
Figure 1. Model of intervention effects on perceived stress, including hypothesized and significant paths

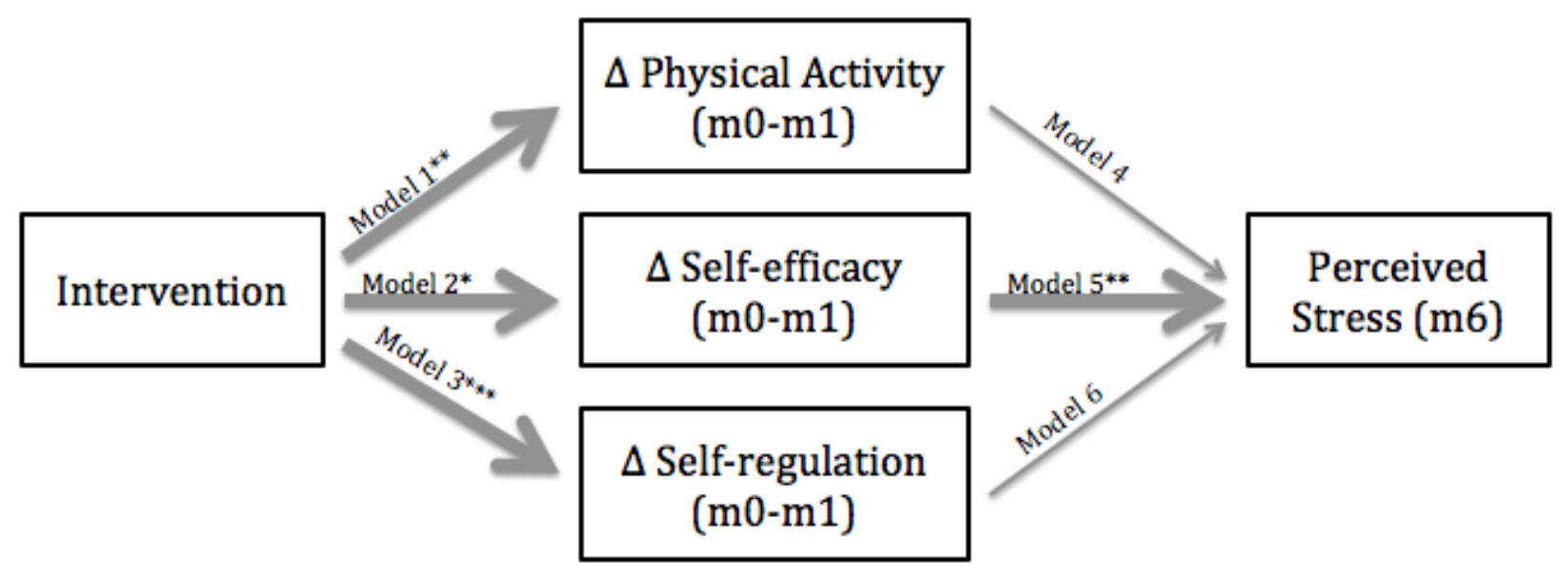

Note. Significant paths are highlighted in bold. ${ }^{*} \mathrm{p}<.05 * * \mathrm{p}<.01 * * * \mathrm{p}<.001$ 


\section{References}

Albright, C. L., J. E. Maddock, and C. R. Nigg. 2005. Physical activity before pregnancy and following childbirth in a multiethnic sample of healthy women in Hawaii. Women Health 42:95110.

Anderson, R. T., A. King, A. L. Stewart, F. Camacho, and W. J. Rejeski. 2005. Physical activity counseling in primary care and patient well-being: Do patients benefit? Ann Behav Med 30:146154.

Bandura, A. 1982. Self-efficacy mechanism in human agency. Am Psychol 37:122-147.

Bandura, A. 1998. Health promotion from the perspective of social cognitive theory. Psychol Health 13:623-649.

Bekker, M. H. J., P. F. de Jong, F. R. H. Zijlstra, and B. A. J. van Landeghem. 2000. Combining care and work: Health and stress effects in male and female academics. Int J Behav Med 7:28-43. Breus, M. J., and P. J. O'Connor. 1998. Exercise-induced anxiolysis: A test of the "time-out" hypothesis in high anxious females. Med Sci Sports Exerc 30:1107-1112.

Brown, P.R., W. J. Brown, Y. D. Miller, and V. Hansen. 2001. Perceived constraints and social support for active leisure among mothers with young children. Leisure Sci 23:131-144.

Bureau of Labor Statistics: Married parents' use of time summary. [http://www.bls.gov/news.release/atus2.nr0.htm] Accessed July 10, 2013.

Burke, V., L. J. Beilin, D. Dunbar, and M. Kevan. 2004. Changes in health-related behaviours and cardiovascular risk factors in young adults: Associations with living with a partner. Prev Med 39:722-730.

Chwalisz, K., E. M. Altmaier, and D. W. Russell. 1992. Causal attributions, self-efficacy cognitions, and coping with stress. J Soc Clin Psychol 11:377-400. 
Cody, R., and C. Lee. 1999. Development and evaluation of a pilot program to promote exercise among mothers of preschool children. Int J Behav Med 6:13-29.

Cohen, S., T. Kamarck, and R. Mermelstein. 1982. A global measure of perceived stress. $J$ Health Soc Behav 24:385-396.

Connell, C. M., and M. R. Janevic. 2009. Effects of a telephone-based exercise intervention for dementia caregiving wives: A randomized controlled trial. J Appl Gerontol 28:171-194.

Cramp, A. G., and L. R. Brawley. 2006. Moms in motion: A group-mediated cognitive behavioral physical activity intervention. Int J Behav Nutr Phys Act 3:23.

Cramp, A. G., and S. R. Bray. 2011. Understanding exercise self-efficacy and barriers to leisuretime physical activity among postnatal women. Matern Child Health J 15:642-651.

Elavsky, S., E. McAuley, R. W. Motl, J. F. Konopack, D. X. Marquez, L. Hu, G. J. Jerome, and E. Diener. 2005. Physical activity enhances long-term quality of life in older adults: Efficacy, esteem, and affective influences. Ann Behav Med 30:138-145.

Fahrenwald, N. L., J. R. Atwood, S. N. Walker, D. R. Johnson, and K. Berg. 2004. A randomized pilot test of "Moms on the Move": A physical activity intervention for WIC mothers. Ann Behav Med 27:82-90.

Fjeldsoe, B. S., Y. D. Miller, and A. L. Marshall. 2010. MobileMums: A randomized controlled trial of an SMS-based physical activity intervention. Ann Behav Med 39:101-111.

Frankenhaeuser, M., U. Lundberg, M. Fredrikson, B. Melin, M. Tuomisto, and A. Myrsten. 1989. Stress on and off the job as related to sex and occupational status in white-collar workers. J Organ Behav 10:321-346.

Godin, G., and R. J. Shephard. 1985. A simple method to assess exercise behavior in the community. Can J Appl Sport Sci 10:141-146. 
Hansen, A. M., Blangsted, A. K., Hansen, E. A., Sogaard, K., and G. Sjogaard. 2010. Physical activity, job demand-control, perceived stress-energy, and salivary cortisol in white-collar workers. Int Arch Occup Environ Health 83:143-153.

Jacobs, D. R., B. E. Ainsworth, T. J. Hartman, and A. S. Leon. 1993. A simultaneous evaluation of 10 commonly used physical activity questionnaires. Med Sci Sports Exerc 25:81-91.

Jarvekulg, A., and A. Viru. 2002. Opioid receptor blockade eliminates mood effects of aerobic gymnastics. Int J Sports Med 23,155-157.

Larzelere, M. M., and G. N. Jones. 2008. Stress and health. Prim Care 35:839-856.

Leucken, L. J., E. C. Suarez, C. M. Kuhn, J. C. Barefoot, J. A. Blumenthal, I. C. Siegler, and R. B. William. 1997. Stress in employed women: Impact of marital status and children at home on neurohormone output and home strain. Psychosom Med 59:352-359.

Long, B. C., and C. J. Haney. 1988. Long-term follow-up of stressed working women: A comparison of aerobic exercise and progressive relaxation. J Sport Exercise Psy 10:461-470.

Lorig, K. R., P. L. Ritter, D. D. Laurent, and K. Plant. 2006. Internet-based chronic disease selfmanagement: A randomized trial. Med Care 44:964-971.

Mailey, E. L., and E. McAuley. 2013. Impact of a brief exercise intervention on physical activity and social cognitive determinants among working mothers: A randomized trial. J Behav Med.

DOI: $10.1007 / \mathrm{s} 10865-013-9492-y$

McAuley, E. 1992. The role of efficacy cognitions in the prediction of exercise behavior in middle-aged adults. J Behav Med 15:65-88.

McAuley, E., S. E. Doerksen, K. S. Morris, R. W. Motl, L. Hu, T. R. Wojcicki, S. M. White, and K. R. Rosengren. 2008. Pathways from physical activity to quality of life in older women. Ann Behav Med 36:13-20. 
McAuley, E., E. L. Mailey, S. P. Mullen, A. N. Szabo, T. R. Wojcicki, S. M. White, N. Gothe, E. A. Olson, and A. F. Kramer. 2011. Growth trajectories of exercise self-efficacy in older adults: Influence of measures and initial status. Health Psychol 30:75-83.

McAuley, E., K. S. Morris, S. E. Doerksen, R. W. Motl, L. Hu, S. M. White, T. R. Wojcicki, and K. R. Rosengren. 2007. Effects of change in physical activity on physical function limitations in older women: Mediating roles of physical function performance and self-efficacy. J Am Geriatr Soc 55:1967-1973.

Miller, Y. D., S. G. Trost, and W. J. Brown. 2002. Mediators of physical activity behavior change among women with young children. Am J Prev Med 23:98-103.

Mohr, D. C., B. Spring, K. E. Freedland, V. Beckner, P. Arean, S. D. Hollon, J. Ockene, and R. Kaplan. 2009. The selection and design of control conditions for randomized controlled trials of psychological interventions. Psychother Psychosom 78:275-284.

Rosenbaum, M., and E. Cohen. 1999. Equalitarian marriages, spousal support, resourcefulness, and psychological distress among Israeli working women. J Vocat Behav 54:102-113.

Rovniak, L. S., E. S. Anderson, R. A. Winett, and R. S. Stephens. 2002. Social cognitive determinants of physical activity in young adults: A prospective structural equation analysis. Ann Behav Med 24:149-156.

Schnohr, P., T. S. Kristensen, E. Prescott, and H. Scharling. 2005. Stress and life dissatisfaction are inversely associated with jogging and other types of physical activity in leisure time - The Copenhagen City Heart Study. Scand J Med Sci Sports 15:107-112.

Tinger, H., G. Kiger, and P. J. Riley. 1996. Juggling multiple roles: Perceptions of working mothers. Soc Sci J 33:183-191. 
Urizar, G. G., S. Q. Hurtz, D. K. Ahn, A. C. King, C. L. Albright, and A. A. Atienza. 2005. Influence of maternal stress on successful participation in a physical activity intervention: The IMPACT Project. Women Health 42:63-82.

Verhoef, M. J., and E. J. Love. 1994. Women and exercise participation: The mixed blessings of motherhood. Health Care Women Int 15:297-306. 